Using an intensified infection prevention intervention to control carbapenemase-producing Enterobacteriaceae at a Thai center. Infect Control Hosp Epidemiol 2012;33:960-961.

6. Siegel JD, Rhinehart E, Jackson M, Chiarello L; Healthcare Infection Control Practices Advisory Committee. Management of multidrug-resistant organisms in health care settings, 2006. Am $J$ Infect Control 2007;35(10 suppl 2):S165-S193.

7. Centers for Disease Control and Prevention (CDC). Guidance for control of infections with carbapenem-resistant or carbapenemase-producing Enterobacteriaceae in acute care facilities. MMWR Morb Mortal Wkly Rep 2009;58:256-260.

\section{Using a Qualitative Study to Understand the Failure of a Strategy Implemented for Improving Hand Hygiene Adherence in 4 Intensive Care Units}

To the Editor-The strategy of active detection and isolation of patients with methicillin-resistant Staphylococcus aureus (MRSA) infection has a number of adverse unintended consequences ${ }^{1}$ and raises ethical concerns. ${ }^{2}$ Concurrently, certain studies have questioned the effectiveness of associating the screening of carriers and the implementation of contact precautions. ${ }^{3,4}$ Although there are some arguments to support a policy of strict application of standard precautions to control MRSA, the low compliance with hand hygiene reported in most studies constitutes what is probably a major barrier against the effectiveness of this policy.,

In a recent study, ${ }^{7}$ we aimed to assess the impact of screening and signaling MRSA carriers on hand hygiene compliance in 4 intensive care units (ICUs) using a strategy of strict application of standard precautions. Although an active campaign of information about MRSA and the potential consequences of MRSA transmission had been performed by the professionals of the infection control (IC) unit of the hospital before this evaluation study, a total absence of impact of the signalization of MRSA carriers on compliance with hand hygiene was recorded.

Concurrent with this study, we performed a qualitative study in the same units, the objective of which was to better understand the feelings of caregivers about IC in general and hand hygiene in particular. The aim of this article is to try to explain the failure of the strategy implemented by using the results of this qualitative study.

This qualitative study included 2 successive phases: a phase of participative observations and a phase of face-to-face interviews. The first phase was conducted during a 4-week period by a master's student in sociology. It is noteworthy that this student was also a registered nurse who did not work in this hospital but concurrently worked in the community setting. She was immersed in the ICUs to observe practices and to talk with healthcare providers, and she also participated in providing patient care. She was in contact with 20-25 healthcare workers each day except weekends between 8 AM and 5 PM. Overall, she met more than 100 healthcare workers who belonged to all categories of personnel. She made a note of her observations and discussions.

Ten face-to-face interviews were conducted. These interviews took 45-60 minutes and were led by 1 of the 2 interviewers who participated in the study: a professor of sociology and the master's student in sociology. All interviews were conducted in accordance with a semistructured interview guide, recorded with a voice recorder, and fully transcribed. The result analysis was performed on the basis of the participative observations and face-to-face interviews.

Most of the caregivers considered IC to be an essential aspect of their work. However, they also mentioned that the transmission of recommendations by IC professionals usually had a weak impact on their practices. The terms "normally," "theoretically," and "in principle" were widely employed by the participants when the protocols and recommendations provided by the IC unit were considered during the interviews. Therefore, these protocols and recommendations seem to have only a relative value. Throughout the conversations with the ICU professionals, the words "we" and "they" were used to refer to ICU staff and the IC professionals, respectively, potentially indicating that the latter were regarded as outsiders whose advice and recommendations were considered an imposition or intrusion upon the culture of the ICU.

In addition, according to the participants, IC is not the only aspect that should be considered during patient care. Other considerations, such as the emergency linked to a clinical situation, can represent a barrier to compliance with good hygiene practices. The desire to protect the relationship between the healthcare professional and the patient can be another barrier. For instance, healthcare workers often expressed concern that wearing gloves, a mask, or goggles might be perceived as hostile to their patients.

Participants reported being more vigilant about prevention measures after contact with patients in situations considered to be physically dirty or "emotionally dirty." In addition, some participants acknowledged that their main motivation for practicing hand hygiene after contact with a patient was to protect themselves. Therefore, it is probable that subjective criteria and self-protective attitudes have a stronger impact on the behavior of healthcare professionals than do recommendations transmitted by the IC unit.

To our knowledge, few qualitative studies have been used to assess the results of a study evaluating a strategy implemented to decrease the risk of cross transmission. Our findings are consistent with the results of some other previous studies. According to Larson et $\mathrm{al}^{8}{ }^{8}$ the diffusion of recommendations from the Centers for Disease Control and Prevention in healthcare settings was not sufficient to change practices. In a recent study, ${ }^{9}$ participants also reported that 
the realities of their workload made complete adherence to hand hygiene impossible, because other patient care needs should be prioritized over hand hygiene. Participants clearly cited personal safety as the primary reason for hand hygiene compliance. Concurrently, it has been demonstrated that risk assessment is influenced by individual differences, including differences in knowledge level and in how much control persons believe that they have over their health. ${ }^{10}$

In conclusion, beliefs and preexisting knowledge probably had a major role in the failure of our strategy. Urgent care, interruptions of care, self-protective behaviors, and subjective assessment of risk could also interfere with the association between hand hygiene compliance and MRSA carriage. This study underscores that, when envisaging IC measures and the evaluation of their impact, it is important to consider that providing healthcare involves not just a collection of duties but also a social relationship.

\section{ACKNOWLEDGMENTS}

We are very grateful to all of the personnel of the 4 ICUs who participated in the study.

Financial support. Interregional Committee for Infection Control in Western France (Centre de Coordination de Lutte contre les Infectious Nosocomiales Ouest, Centre Hospitalier Universitaire, Hôtel Dieu, CS26419, 35064 Rennes Cedex 2).

Potential conflicts of interest. All authors report no conflicts of interest relevant to this article. All authors submitted the ICMJE Form for Disclosure of Potential Conflicts of Interest, and the conflicts that the editors consider relevant to this article are disclosed here.

\section{Matthieu Eveillard, PharmD, PhD; ${ }^{1,2}$ Tiphaine Bruna, RN, MSc; ${ }^{3}$ Achille Kouatchet, MD; ${ }^{4}$ Laurent Dubé, MD; ${ }^{5}$ Laurent Poiroux, RN; ${ }^{4}$ Evelyne Dabin, RN; ${ }^{5}$ Hikombo Hitoto, MD, MPH; ${ }^{1}$ Alain Mercat, $\mathrm{MD}^{4}$ Marie-Laure Joly-Guillou, $\mathrm{MD}, \mathrm{PhD}^{1,2}$ Hélène Desfontaines, $\mathrm{PhD}^{3}$}

Affiliations: 1. Laboratoire de Bactériologie-Hygiène, Centre Hospitalier Universitaire, Angers, France; 2. Groupe d'Etude des Interactions Hôtes Pathogènes, Université d'Angers, Angers, France; 3. Institut de Psychologie et de Sociologie Appliquées, Université Catholique de l'Ouest, Angers, France; 4. Service de Réanimation Médicale, Centre Hospitalier Universitaire, An- gers, France; 5. Service de Réanimation Chirurgicale A, Centre Hospitalier Universitaire, Angers, France.

Address correspondence to Matthieu Eveillard, MD, Laboratoire de Bactériologie-Hygiène, Institut de Biologie en Santé, 4 rue Larrey, F49000 Angers, France (maeveillard@chu-angers.fr).

Infect Control Hosp Epidemiol 2013;34(4):447-448

(C) 2013 by The Society for Healthcare Epidemiology of America. All rights reserved. 0899-823X/2013/3404-0023\$15.00. DOI: 10.1086/669875

\section{REFEREN CES}

1. Diekema DJ, Edmond MB. Look before you leap: active surveillance for multidrug-resistant organisms. Clin Infect Dis 2007; 44:1101-1107.

2. Edmond MB, Lyckholm L, Diekema DJ. Ethical implications of active surveillance culture and contact precautions for controlling multidrug resistant organisms in the hospital setting. Public Health Ethics 2008;1:235-245.

3. Morgan DJ, Diekema DJ, Sepkowitz K, Perencevich EN. Adverse outcomes associated with contact precautions: a review of the literature. Am I Infect Control 2009;37:85-93.

4. McLemore A, Bearman G, Edmond MB. Effect of contact precautions on wait time from emergency room disposition to inpatient admission. Infect Control Hosp Epidemiol 2011;32:298299.

5. Cepeda JA, Whitehouse $\mathrm{T}$, Cooper $\mathrm{B}$, et al. Isolation of patients in single rooms or cohorts to reduce spread of MRSA in intensive care units: prospective two-centre study. Lancet 2005;365:295304.

6. Nijssen S, Bonten MJ, Weinstein RA. Are active microbiological surveillance and subsequent isolation needed to prevent the spread of methicillin-resistant Staphylococcus aureus? Clin Infect Dis 2005;40:405-409.

7. Hitoto $\mathrm{H}$, Kouatchet $\mathrm{A}$, Dubé $\mathrm{L}$, et al. Impact of screening and identifying methicillin-resistant Staphylococcus aureus carriers on hand hygiene compliance in 4 intensive care units. Am J Infect Control 2011;39:571-576.

8. Larson EL, Quiros D, Lin SX. Dissemination of the CDC's hand hygiene guideline and impact on infection rates. Am I Infect Control 2007;35:666-675.

9. Jang $\mathrm{JH}, \mathrm{Wu} S$, Kirzner $\mathrm{D}$, et al. Focus group study of hand hygiene practice among healthcare workers in a teaching hospital in Toronto, Canada. Infect Control Hosp Epidemiol 2010;31:144150.

10. Collins McLaughlin A, Walsh F. Individual differences in judgements of hand hygiene risk by health care workers. Am J Infect Control 2011;39:456-463. 